\title{
Exploring the Degradation Behavior of Ce-Monazite in Water Solution Through Adsorption and Penetration Kinetics
}

\author{
XiaoFeng Zhao ${ }^{\mathrm{a}, \mathrm{b}, \mathrm{c}}$, XiaoYong Yang a,c, YuXiang $\mathrm{Li}^{\mathrm{a}}{ }^{\mathrm{a} b}$ *, Rajeev Ahuja ${ }^{\mathrm{c}, \mathrm{d}}$ *
}

${ }^{a}$ State Key Laboratory for Environment-friendly Energy Materials, Southwest University of Science and Technology, Mianyang 621010, PR China

${ }^{b}$ School of Materials Science and Engineering, Southwest University of Science and Technology, Mianyang 621010, PR China

${ }^{c}$ Condensed Matter Theory Group, Materials Theory Division, Department of Physics and Astronomy, Uppsala University, 75120 Uppsala, Sweden

${ }^{d}$ Applied Materials Physics, Department of Materials and Engineering, Royal Institute of Technology (KTH), S-100 44 Stockholm, Sweden

*Corresponding Author: liyuxiang@swust.edu.cn; rajeev.ahuja@physics.uu.se 


\section{Results and discussion of calculation methods}

The calculated structural property of $\mathrm{CePO}_{4}$ monazite is presented in Table $\mathrm{S} 2$ with $\mathrm{PBE}+\mathrm{U}$ and HSE06 functionals. Notice that the obtained cell volumes by PBE with D2 correction range from $299.236 \AA^{3}$ to $299.295 \AA^{3}$, which is quite consistent with experiment results within less than $1 \%$ error $^{2}$. Due to strong self-interaction electrons in $f$ orbitals of Ce, PBE functional is clearly not able to describe the electronic structure correctly. This can be seen from severely underestimated the band gap (3.56 eV) as compared with experiment value of $5.28 \mathrm{eV}^{3}$. Thus, an appropriate $\mathrm{U}$ value has been used in our work to correct the strong self-interaction electrons. Considering a series of $\mathrm{U}$ parameters from $5.5 \mathrm{eV}$ to $6.5 \mathrm{eV}$, the most appropriate $\mathrm{U}$ value is $6 \mathrm{eV}$ corresponding to the band gap of $5.32 \mathrm{eV}$. Besides, the DOS features of PBE+U $(6 \mathrm{eV})$ is similar to the evolution of HSE06, as shown in Fig. S1.

Fig. S1 shows the structure of monoclinic $\mathrm{CePO}_{4}$ monazite. Its pristine lattice is composed of four structural units, each containing one $\mathrm{Ce}$ atom and one $\left[\mathrm{PO}_{4}\right]$ tetrahedron. The tetrahedron is isolated and separated by $\left[\mathrm{CeO}_{9}\right]$ polyhedron, and each $\mathrm{O}$ atom coordinate to one $\mathrm{P}$ and two $\mathrm{Ce}$ atoms ${ }^{4,5}$. The structural arrangement in $\mathrm{CePO}_{4}$ crystallographic system is based on $\left[\mathrm{CeO}_{9}\right]$ polyhedron interpenetrated by a $\left[\mathrm{PO}_{4}\right]$ tetrahedron to form infinite chains along the $\mathrm{c}$ axis, as shown in Fig. 1(b). Clearly, adjacent chains are different principally in element coordinates, resulting in discrete distribution of nine $\mathrm{O}$ atoms around $\mathrm{Ce}$ atoms and then forming the irregular $\left[\mathrm{CeO}_{9}\right]$ polyhedron.

Furthermore, we used the Baur's distortion index $(\Delta)$ to quantitatively describe the structural distortion of $\left[\mathrm{CeO}_{9}\right]$ polyhedron and $\left[\mathrm{PO}_{4}\right]$ tetrahedron affected by exchange correlation functional, which is expressed as ${ }^{6}$ :

$$
\Delta(\mathrm{X}-0)=\frac{1}{\mathrm{n}} \sum_{\mathrm{i}=1}^{\mathrm{n}} \frac{\left|(\mathrm{X}-0)_{\mathrm{i}}-\langle\mathrm{X}-0\rangle\right|}{\langle\mathrm{X}-0\rangle}
$$

where $\mathrm{X}$ is $\mathrm{Ce}$ or $\mathrm{P}$, and its coordination number is represented by $\mathrm{n} ;(\mathrm{X}-\mathrm{O})$ and $\langle\mathrm{X}-\mathrm{O}\rangle$ are the bond length and average bond length between $\mathrm{M}$ and $\mathrm{O}$ atoms, respectively. With the reference value corresponding to experiment results, it clearly shows that the van der Waals correction term can slightly increase the distortion of $\left[\mathrm{CeO}_{9}\right]$ polyhedron and $\left[\mathrm{PO}_{4}\right]$ tetrahedron by overestimated $\Delta$ values. Moreover, $\mathrm{U}$ values shows a neglectable influence on the local structure of $\left[\mathrm{CeO}_{9}\right]$ polyhedron and $\left[\mathrm{PO}_{4}\right]$ tetrahedron. 


\section{Tables}

\section{Table S1}

Defining the boundary constrains for crystal-growing of $\mathrm{CePO}_{4}$ with avoiding the precipitation of undesirable phases. The formation enthalpies $\left(\Delta E^{\mathrm{f}}, \mathrm{eV}\right)$ from calculation and experiment ${ }^{1}$ are also listed in this table.

\begin{tabular}{|c|c|c|c|}
\hline $\begin{array}{l}\text { Expected } \\
\text { compounds }\end{array}$ & Stability condition & $\Delta E^{\mathrm{f}}($ cal. $)$ & $\Delta E^{\mathrm{f}}(\exp .)^{1}$ \\
\hline $\mathrm{CePO}_{4}$ & $\Delta \mu_{\mathrm{Ce}}+\Delta \mu_{\mathrm{P}}+4 \Delta \mu_{0}=\Delta \mathrm{E}_{\mathrm{CePO}_{4}}^{\mathrm{f}}$ & -2.820 & -3.410 \\
\hline $\begin{array}{l}\text { Competing } \\
\text { compounds }\end{array}$ & Boundary constrains & $\Delta E^{\mathrm{f}}(\mathrm{cal})$. & $\Delta E^{\mathrm{f}}($ exp.) \\
\hline $\mathrm{O}_{2}$ & $\Delta \mu_{0}<0$ & - & - \\
\hline $\mathrm{Ce}$ & $\Delta \mu_{\mathrm{Ce}}<0$ & - & - \\
\hline $\mathrm{P}$ & $\Delta \mu_{\mathrm{O}}>1 / 4\left(\Delta \mathrm{E}_{\mathrm{CePO}_{4}}^{\mathrm{f}}-\Delta \mu_{\mathrm{Ce}}\right)$ & - & - \\
\hline $\mathrm{PO}_{2}$ & $\Delta \mu_{\mathrm{O}}=1 / 2\left(\Delta \mathrm{E}_{\mathrm{CePO}_{4}}^{\mathrm{f}}-\Delta \mathrm{E}_{\mathrm{PO}_{2}}^{\mathrm{f}}-\Delta \mu_{\mathrm{Ce}}\right)$ & -1.792 & -1.625 \\
\hline $\mathrm{P}_{2} \mathrm{O}_{5}$ & $\Delta \mu_{0}>1 / 3\left(2 \Delta \mathrm{E}_{\mathrm{CePO}_{4}}^{\mathrm{f}}-\Delta \mathrm{E}_{\mathrm{P}_{2} \mathrm{O}_{5}}^{\mathrm{f}}-2 \Delta \mu_{\mathrm{Ce}}\right)$ & -1.542 & -2.461 \\
\hline $\mathrm{P}_{2} \mathrm{O}_{3}$ & $\Delta \mu_{0}=1 / 5\left(2 \Delta \mathrm{E}_{\mathrm{CePO}_{4}}^{\mathrm{f}}-\Delta \mathrm{E}_{\mathrm{P}_{2} \mathrm{O}_{3}}^{\mathrm{f}}-2 \Delta \mu_{\mathrm{Ce}}\right)$ & -2.214 & -1.976 \\
\hline $\mathrm{CeO}$ & $\Delta \mu_{0}=\Delta \mathrm{E}_{\mathrm{CeO}}^{\mathrm{f}}-\Delta \mu_{\mathrm{Ce}}$ & -3.001 & -3.246 \\
\hline $\mathrm{CeO}_{2}$ & $\Delta \mu_{0}=1 / 4\left(\Delta \mathrm{E}_{\mathrm{CeO}_{2}}^{\mathrm{f}}-\Delta \mu_{\mathrm{Ce}}\right)$ & -3.548 & -3.950 \\
\hline $\mathrm{Ce}_{2} \mathrm{O}_{3}$ & $\Delta \mu_{0}<1 / 3\left(\Delta \mathrm{E}_{\mathrm{Ce}_{2} \mathrm{O}_{3}}^{\mathrm{f}}-2 \Delta \mu_{\mathrm{Ce}}\right)$ & -3.395 & -3.781 \\
\hline $\mathrm{CeP}_{2} \mathrm{O}_{7}$ & $\Delta \mu_{0}<1 / 6\left(\Delta \mathrm{E}_{\mathrm{CeP}_{2} \mathrm{O}_{7}}^{\mathrm{f}}-2 \Delta \mathrm{E}_{\mathrm{CePO}_{4}}^{\mathrm{f}}\right)$ & -2.631 & -3.132 \\
\hline $\mathrm{CeP}_{5} \mathrm{O}_{14}$ & $\Delta \mu_{0}>1 / 6\left(5 \Delta \mathrm{E}_{\mathrm{CePO}_{4}}^{\mathrm{f}}-\Delta \mathrm{E}_{\mathrm{CeP}_{5} \mathrm{O}_{14}}^{\mathrm{f}}-4 \Delta \mu_{\mathrm{Ce}}\right)$ & -2.186 & -2.825 \\
\hline $\mathrm{Ce}\left(\mathrm{PO}_{3}\right)_{3}$ & $\Delta \mu_{0}>1 / 3\left(3 \Delta \mathrm{E}_{\mathrm{CePO}_{4}}^{\mathrm{f}}-\Delta \mathrm{E}_{\mathrm{Ce}\left(\mathrm{PO}_{3}\right)_{3}}^{\mathrm{f}}-2 \Delta \mu_{\mathrm{Ce}}\right)$ & -2.480 & -2.980 \\
\hline
\end{tabular}




\section{Table S2}

The lattice parameters $(\AA)$, cell volume $\left(\AA^{3}\right)$, average bond distance $\langle\mathrm{Ce} / \mathrm{P}-\mathrm{O}\rangle$, and the Baur distortion coefficient $(\Delta)$ of $\mathrm{CePO}_{4}$ monazite calculated by $\mathrm{PBE}+\mathrm{U}$ with and without vdW correction, and HSE06 functionals. Besides, the experiment results are presented for comparison.

\begin{tabular}{|c|c|c|c|c|c|c|c|c|}
\hline & \multicolumn{6}{|c|}{$\mathrm{PBE}+\mathrm{U}$} & \multirow{2}{*}{ HSE06 } & \multirow{2}{*}{ Exp. } \\
\hline & $\mathrm{U}=0$ & $\mathrm{U}=6$ & $\mathrm{U}=0, \mathrm{D} 2$ & $\mathrm{U}=5.5, \mathrm{D} 2$ & $\mathrm{U}=6, \mathrm{D} 2$ & $\mathrm{U}=6.5, \mathrm{D} 2$ & & \\
\hline$a$ & 6.880 & 6.880 & 6.706 & 6.706 & 6.707 & 6.706 & 6.775 & 6.797 \\
\hline$b$ & 7.140 & 7.140 & 6.964 & 6.965 & 6.965 & 6.966 & 7.018 & 7.022 \\
\hline$c$ & 6.542 & 6.542 & 6.567 & 6.567 & 6.567 & 6.567 & 6.449 & 6.472 \\
\hline$\beta$ & 103.226 & 103.224 & 102.660 & 102.661 & 102.670 & 102.675 & 103.305 & 103.466 \\
\hline V & 312.844 & 312.845 & 299.236 & 299.290 & 299.271 & 299.295 & 298.402 & 300.458 \\
\hline$\langle\mathrm{Ce}-\mathrm{O}\rangle$ & 2.593 & 2.593 & 2.562 & 2.562 & 2.562 & 2.562 & 2.551 & 2.557 \\
\hline$\Delta(\mathrm{Ce}-\mathrm{O})$ & 0.033 & 0.033 & 0.036 & 0.037 & 0.037 & 0.037 & 0.034 & 0.036 \\
\hline$\langle\mathrm{P}-\mathrm{O}\rangle$ & 1.553 & 1.554 & 1.547 & 1.547 & 1.547 & 1.547 & 1.533 & 1.556 \\
\hline$\Delta(\mathrm{P}-\mathrm{O})$ & 0.007 & 0.007 & 0.008 & 0.009 & 0.009 & 0.009 & 0.006 & 0.007 \\
\hline
\end{tabular}




\section{Table S3}

The surface energy of different $\mathrm{CePO}_{4}$ surfaces

\begin{tabular}{cccccc}
\hline Surface & $(100)$ & $(010)$ & $(001)$ & $(121)$ & $(110)$ \\
\hline $\begin{array}{c}\mathrm{E}_{\text {surf }} \\
\left(\mathrm{J} / \mathrm{m}^{2}\right)\end{array}$ & $1.45(5)$ & $1.45(4)$ & $2.59(2)$ & $1.60(0)$ & $1.48(6)$ \\
\hline
\end{tabular}

Table S4

The charge transfer of the $\mathrm{CePO}_{4}(010)$ surface with adsorbing different coverage of $\mathrm{H}_{2} \mathrm{O}$ molecules

\begin{tabular}{ccccccccc}
\hline$\Delta \mathrm{Q}$ & \multicolumn{8}{c}{$\mathrm{nH}_{2} \mathrm{O}$} \\
\cline { 2 - 9 }$(\mathrm{e})$ & 1 & 2 & 3 & 4 & 5 & 6 & 7 & 8 \\
\hline Total & -0.0312 & -0.0537 & -0.0599 & -0.0549 & -0.0753 & -0.1062 & -0.0851 & -0.0637 \\
average & -0.0312 & -0.2685 & -0.0200 & -0.0137 & -0.0151 & -0.0177 & -0.0122 & -0.0080 \\
\hline
\end{tabular}




\section{Figures}

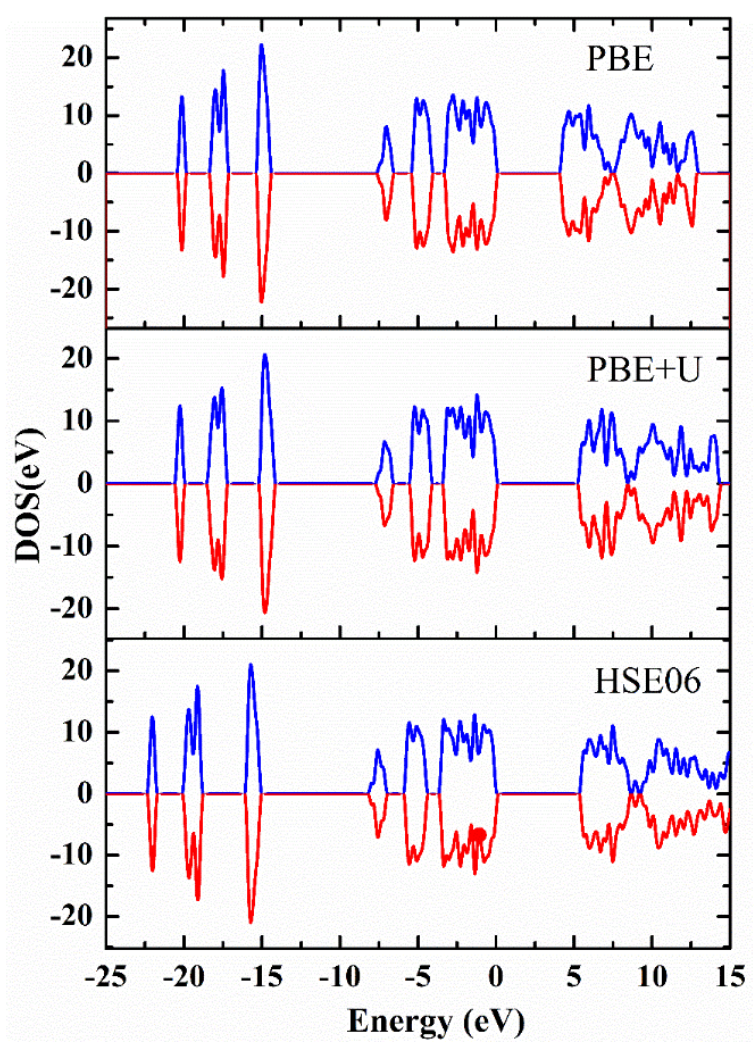

Figure S1 Total-DOS of fully-sodiated structure calculated with PBE, PBE+U (6 eV), and HSE06 exchange correlation functionals. The energies are referenced with the Fermi level (Ef)

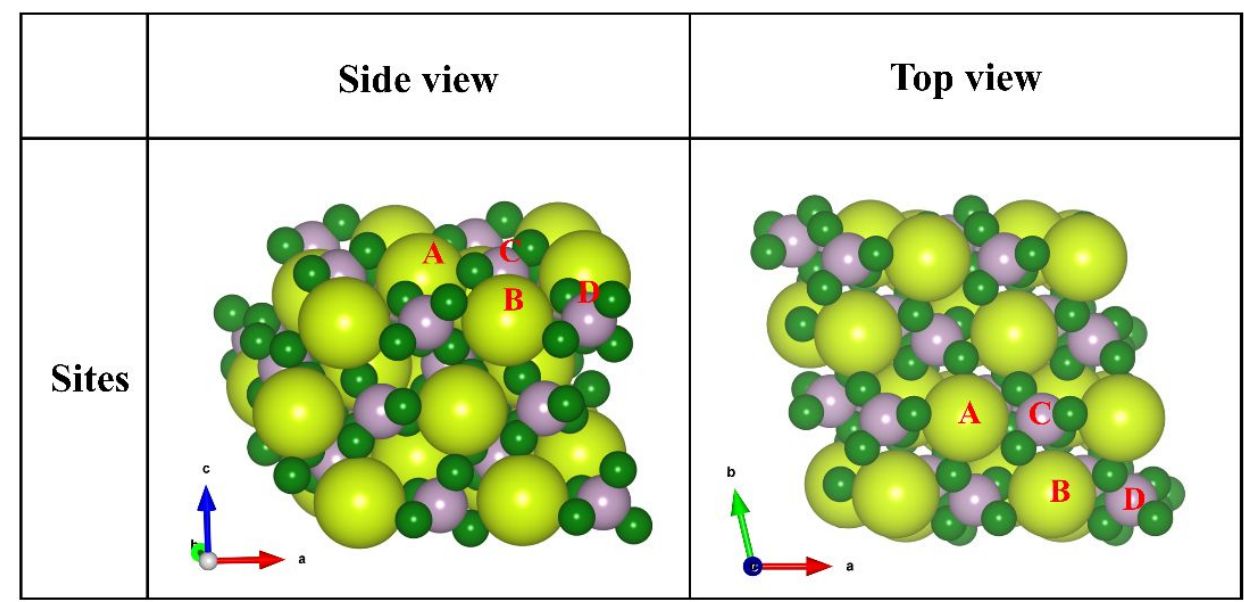

Figure S2 The possible adsorption sites for $\mathrm{H}_{2} \mathrm{O}$ molecule on (010) surface. The (a) and (b) illustrations show the side and top views. 


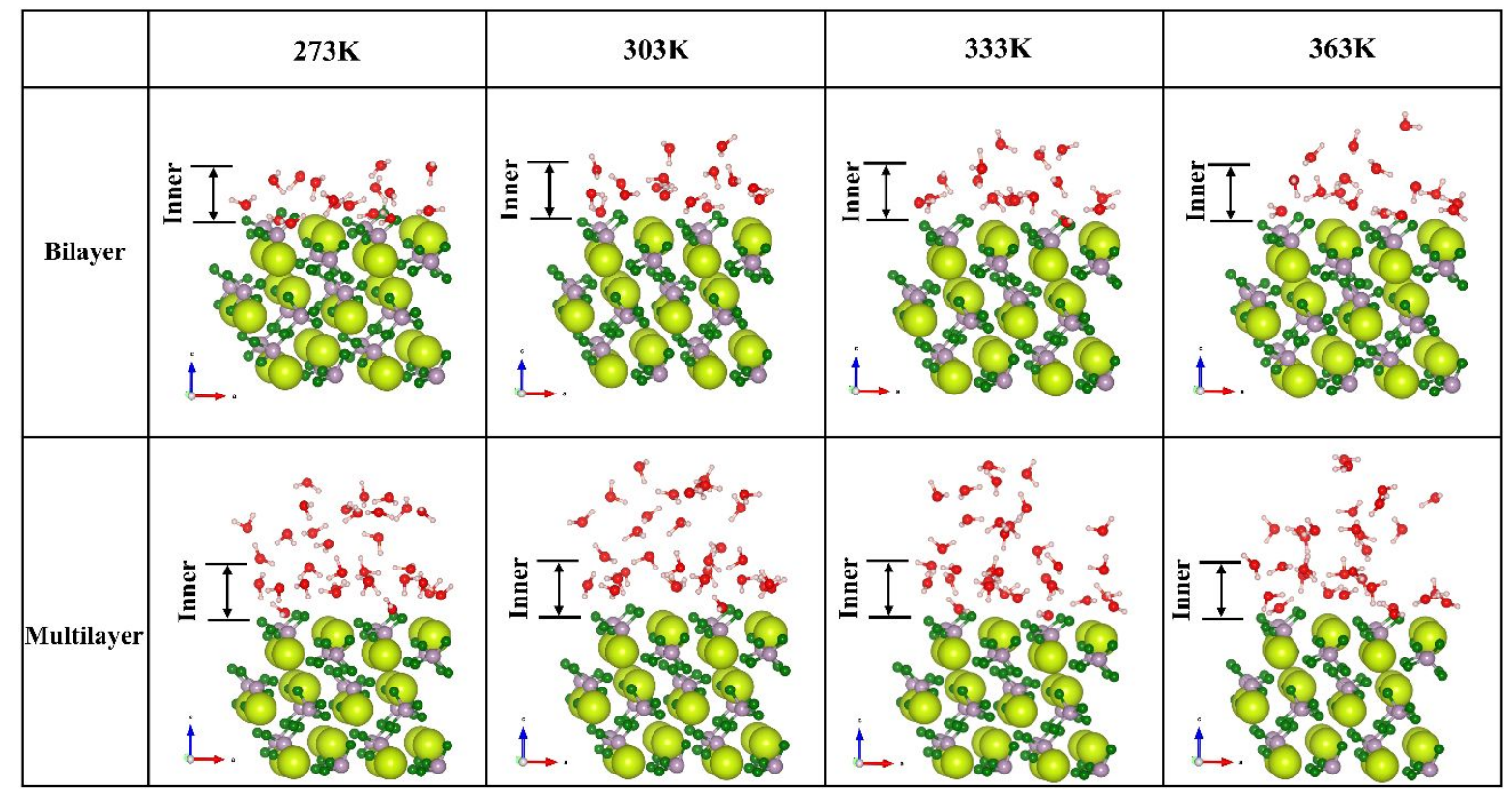

Figure S3 The structures of bilayer and multilayers adsorbed on (010) surface under different temperature.

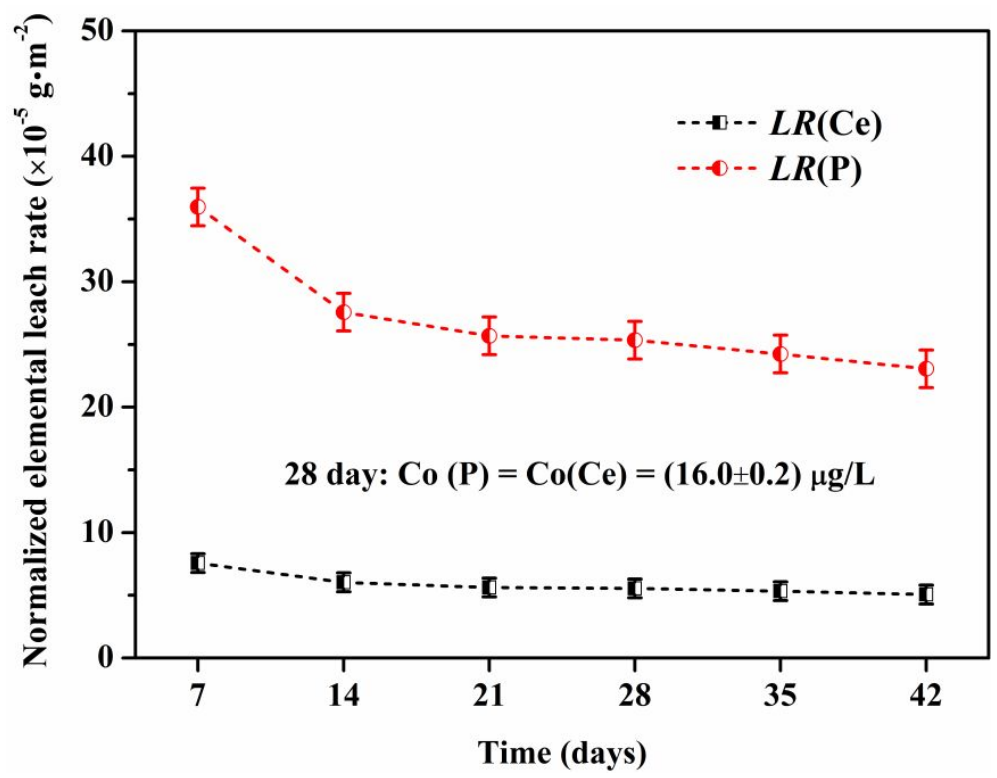

Figure S4 The calculated normalized elemental leach rate of Ce and $\mathrm{P}$ elements for $\mathrm{CePO}_{4}$ monazite after leaching from $7 \mathrm{~d}$ to $42 \mathrm{~d}$ in $0.001 \mathrm{~mol} / \mathrm{L} \mathrm{HCl}$ solution. The experimental methods are from reference $[7]^{7}$. 


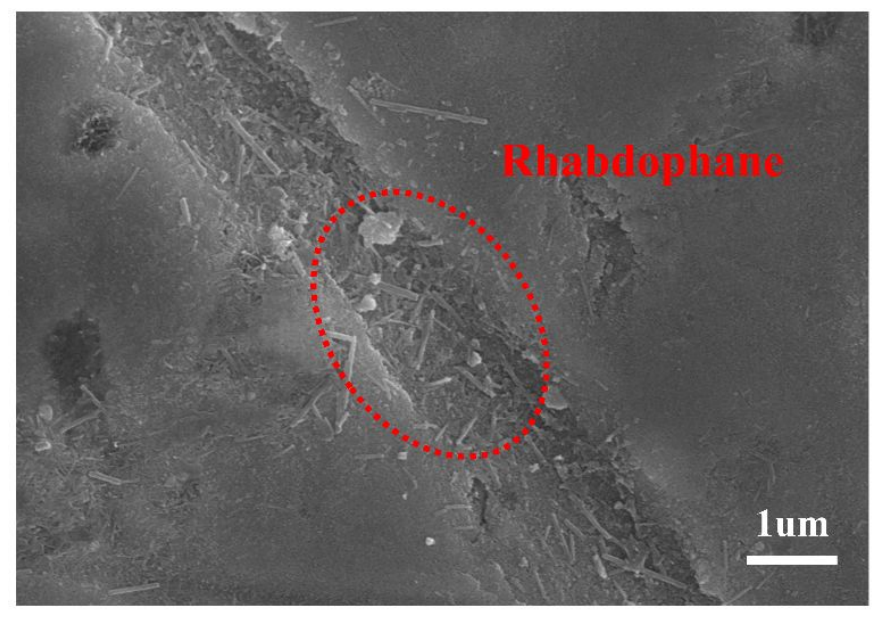

Figure S5 The SEM of $\mathrm{CePO}_{4}$ monazite ceramics after leaching reaction. 


\section{References}

1. A. Jain, S.P. Ong, G. Hautier, W. Chen, W.D. Richards, S. Dacek, S. Cholia, D. Gunter, D. Skinner, G. Ceder, and K.A. Persson, APL Mater. 1, (2013).

${ }^{2}$ X. Zhao, Y. Li, Y. Teng, L. Wu, P. Bi, X. Yang, and L. Wan, J. Eur. Ceram. Soc. 39, 1555 (2019).

${ }^{3}$ M. Kirubanithy, A.A. Irudayaraj, A.D. Raj, and S. Manikandan, Mater. Today Proc. 2, 4344 (2015).

${ }^{4}$ Y. Ni, J.M. Hughes, A.N. Mariano, N.I. Et, and A.L. Crystal, Am. Mineral. 80, 21 (1995).

${ }^{5}$ N. Clavier, R. Podor, and N. Dacheux, J. Eur. Ceram. Soc. 31, 941 (2011).

${ }^{6}$ W.H. Baur, Acta Crystallogr. Sect. B Struct. Crystallogr. Cryst. Chem. 30, 1195 (1974).

7. X. Zhao, Y. Teng, L. Wu, Y. Huang, J. Ma, G. Wang, J. Nucl. Mater. 466 187-193 (2015). 